\title{
Sex Differences of Periaqueductal Grey Matter Functional Connectivity in Migraine
}

\begin{abstract}
Kinga Gecse ${ }^{1,2}$, Daniel Baksa ${ }^{1,2}$, Dóra Dobos ${ }^{1,2}$, Csaba Sandor Aranyi ${ }^{3}$, Attila Galambos ${ }^{2,4}$, Natália Kocsel ${ }^{2,4}$, Edina Szabó ${ }^{2,4,5}$, Gyöngyi Kökönyei ${ }^{1,2,4}$, Miklós Emri ${ }^{3}$, Gyorgy Bagdy 1,6,7 and Gabriella Juhasz ${ }^{1,2 *}$

${ }^{1}$ Department of Pharmacodynamics, Faculty of Pharmacy, Semmelweis University, Budapest, Hungary, ${ }^{2}$ SE-NAP2 Genetic Brain Imaging Migraine Research Group, Hungarian Brain Research Program, Semmelweis University, Budapest, Hungary, ${ }^{3}$ Division of Nuclear Medicine and Translational Imaging, Department of Medical Imaging, Faculty of Medicine, University of Debrecen, Debrecen, Hungary, ${ }^{4}$ Institute of Psychology, ELTE Eötvös Loránd University, Budapest, Hungary, ${ }^{5}$ Center for Pain and the Brain (PAIN Research Group), Department of Anesthesiology, Critical Care and Pain Medicine, Boston Children's Hospital, Harvard Medical School, Boston, MA, United States, ${ }^{6}$ NAP-2-SE New Antidepressant Target Research Group, Hungarian Brain Research Program, Semmelweis University, Budapest, Hungary, ${ }^{7}$ MTA-SE Neuropsychopharmacology and Neurochemistry Research Group, Hungarian Brain Research Program, Semmelweis University, Budapest, Hungary
\end{abstract}

\section{OPEN ACCESS}

Edited by: Nasim Maleki,

Harvard Medical School, United States

Reviewed by:

Marcos Fabio DosSantos, Federal University of Rio de Janeiro, Brazi

Danielle D. DeSouza

Winterlight Labs, Canada

*Correspondence:

Gabriella Juhasz

juhasz.gabriella@ pharma.semme/weis-univ.hu

Specialty section: This article was submitted to Headache,

a section of the journal

Frontiers in Pain Research

Received: 30 August 2021 Accepted: 02 November 2021 Published: 30 November 2021

Citation:

Gecse K, Baksa D, Dobos D, Aranyi CS, Galambos A, Kocsel N, Szabó E, Kökönyei G, Emri M, Bagdy G and Juhasz G (2021) Sex Differences of Periaqueductal Grey Matter Functional Connectivity in Migraine. Front. Pain Res. 2:767162. doi: 10.3389/fpain.2021.767162
The existence of "sex phenotype" in migraine is a long-standing scientific question. Fluctuations of female sex hormones contribute to migraine attacks, and women also have enhanced brain activity during emotional processing and their functional brain networks seem to be more vulnerable to migraine-induced disruption compared to men. Periaqueductal grey matter (PAG) is a core region of pain processing and modulation networks with possible sex-related implications in migraine. In our study, sex differences of PAG functional resting-state connectivity were investigated in the interictal state in 32 episodic migraines without aura patients (16 women and 16 men). A significant main effect of sex was detected in PAG connectivity with postcentral, precentral, and inferior parietal gyri, and further differences were found between right PAG and visual areas (superior occipital gyrus, calcarine, and cuneus), supplementary motor area, and mid-cingulum connectivity. In all cases, PAG functional connectivity was stronger in female migraineurs compared to males. However, higher average pain intensity of migraine attacks correlated with stronger connectivity of PAG and middle temporal, superior occipital, and parietal gyri in male migraineurs compared to females. Migrainerelated disability is also associated with PAG connectivity but without sex differences. Our results indicate that sex differences in PAG connectivity with brain regions involved in sensory and emotional aspects of pain might contribute to the "sex-phenotype" in migraine. The stronger functional connectivity between PAG and pain processing areas may be a sign of increased excitability of pain pathways even in resting-state in females compared to male migraineurs, which could contribute to female vulnerability for migraine. However, pain intensity experienced by male migraineurs correlated with increased connectivity between PAG and regions involved in the subjective experience of pain and pain-related unpleasantness. The demonstrated sex differences of PAG functional connectivity may support the notion that the female and male brain is differently affected by migraine.

Keywords: headache, periaqueductal grey (PAG), male, female, pain intensity, disability 


\section{INTRODUCTION}

Despite the extensive research in recent decades and successful drug developments, migraine remains one of the major causes of disability worldwide and the first cause among young women (1). Migraine affects women three times more than men suggesting that sex differences, among other factors, take part in migraine attack generation (2). Pain perception and pain-related unpleasantness alter during the menstrual cycle (3), and hormonal fluctuations make women more sensitive to migraine triggers $(4,5)$. Furthermore, perimenopausal women with migraine have a higher allostatic load than non-migraineurs (6) that leads to maladaptive stress responses and reinforces the generation of migraine attacks (7).

In addition to hormonal factors, recent structural and functional imaging studies revealed that the brains of women and men are differentially affected by migraine (8). A meta-analysis reported a decreased grey matter volume in the right dorsolateral prefrontal cortex among female migraineurs (9). Moreover, increased cortical thickness in insula and precuneus was found in female migraineurs compared to males and healthy controls from both sexes (10). In addition, stronger functional connectivity of these two regions with other emotional processing areas (e.g., primary somatosensory cortex) was observed in female migraineurs suggesting that females seem to be more vulnerable to migraine-induced disruption in emotional circuitry (10). Migraine patients showed enhanced somatosensory response to emotional stimuli in a face emotional task indicating an increased sensitivity to psychosocial stressors (11). Disrupted functional connectivity in brain regions involved in sensory processing and pain modulation has been also demonstrated among female migraineurs compared to healthy women (12), but there was no comparison with males. Migraine is considered a typical disease in women and they are overrepresented in migraine research. However, recent findings pointed out the importance of involving men in migraine studies to distinguish male- and female-specific neuropathology $(8,13)$.

A possible candidate region for detecting further cerebral differences between female and male migraineurs is the periaqueductal grey matter (PAG), a core region of a pain processing and modulating network. First, PAG dysfunction contributes to migraine pathogenesis (14), wherein the increased activity of PAG was detected during migraine attacks (15) and altered interictal PAG functional connectivity (PAG-FC), with nociceptive and somatosensory processing pathways, was also found (16). Second, sex differences were revealed in pain-induced PAG connectivity in non-migraineurs (13), specifically, men who had increased PAG-FC with the amygdala, putamen, and caudate compared to women. This result obtained from a pain task may suggest that PAG connectivity may also differ between sexes among migraine patients. Finally, female hormones influence prostaglandin E2 $\left(\mathrm{PGE}_{2}\right)$ and calcitonin gene-related peptide (CGRP) levels in PAG (17), representing a mechanism that could contribute to migraine attack development in women.

Based on the above findings, we hypothesised that PAG functional connectivity may show differences between female and male migraineurs. In addition, we assumed that sex might have an impact on the relationship between PAG connectivity and migraine attack frequency and migraine-related disability. To test this hypothesis, we conducted a resting-state fMRI study with migraine patients.

\section{MATERIALS AND METHODS}

\section{Participants}

Thirty-two age-matched episodic migraine patients without aura were included in the study, 16 men (mean age $=29.73 \pm 6.12$ years) and 16 women (mean age $=29.36 \pm 6.20$ years). To achieve an adequate sample size, data from two studies were pooled together. Participants were pain- and medication-free $48 \mathrm{~h}$ before the examination and did not use any daily medication, except contraceptives. Before entering the study, the mental health of participants was checked by senior researchers using the Mini-International Neuropsychiatric Interview (18) and episodic migraine without aura was diagnosed by expert neurologists using International Classification of Headache Disorders-III criteria (19). Participants did not have any past or current serious medical, neurologic, or psychiatric disorders. They were all righthanded in accordance with the Edinburgh Handedness Inventory (20). On the fMRI examination day, participants completed the Migraine Disability Assessment (MIDAS) questionnaire (21) and were asked about the frequency of migraine attacks per month.

The study protocol was approved by the Scientific and Research Ethics Committee of the Medical Research Council (Hungary). The entire study was conducted according to the Declaration of Helsinki and with the written informed consent of each participant.

\section{Imaging Data Acquisition}

Resting-state fMRI was obtained using two different types of 3 Tesla MRI scanners: half of the participants (eight women and eight men) was scanned with an Achieva $3 \mathrm{~T}$ Philips Medical System (Philips Healthcare, Amsterdam, Netherlands), while the other half of our sample with a $3 \mathrm{~T}$ MAGNETOM Prisma Siemens Syngo (Siemens Healthineers, Erlangen, Germany). The study protocol and the imaging data preprocessing steps were the same in both cases. Participants were required to close their eyes, but remain awake. The imaging dataset acquisition parameters of Philips Achieva T2*-weighted echo-planar (EPI) pulse-sequence were as follows: repetition time $(\mathrm{TR})=2,500 \mathrm{~ms}$, echo time (TE) $=30 \mathrm{~ms}$, field of view $(\mathrm{FOV})=240 \mathrm{~mm}^{2}$; with a $3 \times 3 \times$ $3 \mathrm{~mm}$ resolution. The imaging dataset acquisition parameters of MAGNETOM Prisma were as follows: $\mathrm{TR}=2,220 \mathrm{~ms}$, $\mathrm{TE}=$ $30 \mathrm{~ms}, \mathrm{FOV}=222 \mathrm{~mm}^{2}$, with a $3 \times 3 \times 3 \mathrm{~mm}$ resolution. Highresolution $(1 \times 1 \times 1 \mathrm{~mm})$ structural data were acquired before the resting-state session using a T1-weighted 3D turbo field echo (TFE) sequence with Philips scanner and 3D MPRAGE sequence with Siemens scanner. The possible effect of the scanners was taken into account during the statistical analysis.

\section{Self-Reported Measures}

The first five items of the MIDAS questionnaire (21) were summed and used as a measure of migraine-related disability in the last 3 months before the experiment. The MIDAS-B ("On $a$ 
scale of $0-10$, on average how painful were these headaches?") was used to measure the average pain intensity of migraine attacks. Two participants (one female and one male) did not complete the MIDAS questionnaire.

\section{Data Analysis}

The characteristics and sex differences of participants in selfreported data were analysed using SPSS (IBM Corp., Armonk, NY, USA, SPSS Statistics for Windows, Version 27) with a significance threshold set at $p<0.05$.

\section{Image Preprocessing}

Freesurfer version 5.3 (https://surfer.nmr.mgh.harvard.edu/) (22) was used to perform non-uniformity correction, intensity normalisation, and segmentation of the structural images to extract the brain volume and to separate white matter and grey matter tissues from cerebrospinal fluid (CSF). Spatial normalisation to a $2 \mathrm{~mm}$ isovoxel brain template in MNI152 space (Montreal Neurological Institute, McGill University, Montreal, Canada) (23) was performed with the advanced normalisation tools (ANTs) registration tool (24). Motion correction FMRIB's linear image registration tool (MCFLIRT) (25) utility of the FMRIB's Software Library (FSL version 6 created by the Analysis Group, FMRIB, Oxford, UK) was used for primary motion correction. In the spatial standardisation step, the FMRIB's Software Library (FSL) linear registration tool coregistered the functional images with the structural image of the corresponding subject, then transformed them into MNI152 space. CompCor technique (26) was used to compute the first five principal components of the fMRI timeseries within the white matter and CSF. Spatial filtering was performed with the FSL application SUSAN (27) using a $6 \mathrm{~mm}$ Gaussian kernel. Independent component analysis based on automatic removal of motion artefacts (ICA-AROMA) (28) was used to remove signal components classified as related to motion. For further signal correction, we regressed nuisance variables of 24 movement-related effects computed from rigid body transformation parameters (29), and the five principal components amount to the most variation measured in selected noise regions (white matter and cerebrospinal fluid) (26), which were segmented by Freesurfer. Accounting for white matter and CSF signals is especially important in the investigation of PAG, which is highly influenced by the nearby sources of noise. Finally, temporal band-pass filtering in the range of 0.009 and $0.08 \mathrm{~Hz}$ was applied. The schematic picture of preprocessing steps is provided in the Supplementary Figure 1.

\section{Resting-State fMRI Analysis}

Seed-to-voxel analysis was conducted with left and right PAG as seed regions defined after Mainero et al. (16) (Left PAG = $-2 ;-28 ;-6$; Right PAG $=4 ;-28 ;-6$; coordinates in MNI space with a $3 \mathrm{~mm}$ radius; see Supplementary Figure 2). Seedbased connectivity maps were generated by voxel-wise Pearson correlation with averaged seed region data of each subject and converted into $Z$-scores using Fisher transformation.

These individual connectivity matrices were entered to a second-level full factorial ANOVA using Statistical Parametric Mapping (SPM12) software package (Wellcome Department of Imaging Neuroscience, Institute of Neurology, London, UK; http://www.fil.ion.ucl.ac.uk/spm12/) implemented in Matlab 2016a (Math Works, Natick, MA, USA). An explicit grey matter mask was applied in every analysis for noise-reduction, the template was provided by the Brain Imaging Centre (Montreal Neurological Institute, McGill University: https://digital.lib. washington.edu/researchworks/handle/1773/33312). To be able to evaluate the scanner effect the first factor in the full factorial ANOVA was scanner type. The second factor was sex and we investigated the main effect of the scanner, the main effect of sex, and their potential interaction. To follow up the ANOVA results, post-hoc $t$-tests were performed.

The main effect of migraine disability, average pain intensity, and migraine frequency, and their interaction effect with sex on PAG intrinsic functional connectivity (PAG-FC) were investigated with two-sample $t$-tests.

To investigate the menstrual cycle differences on PAG-FC in female participants, the PAG-FC of females in a luteal phase were compared to PAG-FC of females in the follicular phase using twosample $t$-tests. The effect of oral contraceptives on PAG-FC was also investigated in a two-sample $t$-test comparing the group of females using birth control pills to those who are not using them.

All models were corrected for motion parameters and age. We report peak $T$-values for our analysis in clusters where familywise error corrected $p_{\text {FWE }}<0.05$ significance threshold were reached in at least 20 contiguous voxels (30).

\section{RESULTS}

\section{Participant Characteristics}

The men and women migraine group did not differ in age, migraine frequency, average pain intensity, or MIDAS total score (refer to Table 1), which means that their migraine severity was similar. The number of participants was equally distributed

TABLE 1 | Characteristics of the participants.

\begin{tabular}{|c|c|c|c|c|}
\hline & Male & Female & Test statistics $(U)$ & $p$ \\
\hline Number of participants & 16 & 16 & & \\
\hline Age (years) & $29.73 \pm 6.12$ & $29.36 \pm 6.20$ & 119.5 & 0.747 \\
\hline Migraine frequency / month & $3.34 \pm 2.23$ & $2.80 \pm 3.17$ & 92.5 & 0.176 \\
\hline MIDAS total score & $7.93 \pm 7.42$ & $11.6 \pm 12.32$ & 99.0 & 0.574 \\
\hline Average pain intensity (MIDAS-B) & $5.20 \pm 1.96$ & $5.73 \pm 1.44$ & 99.5 & 0.578 \\
\hline
\end{tabular}

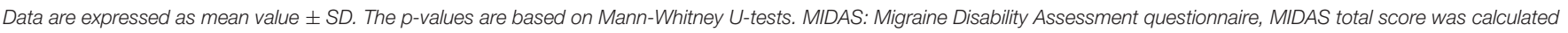
as a measure of migraine-related disability, MIDAS-B refers to the average pain intensity of migraine attacks. 


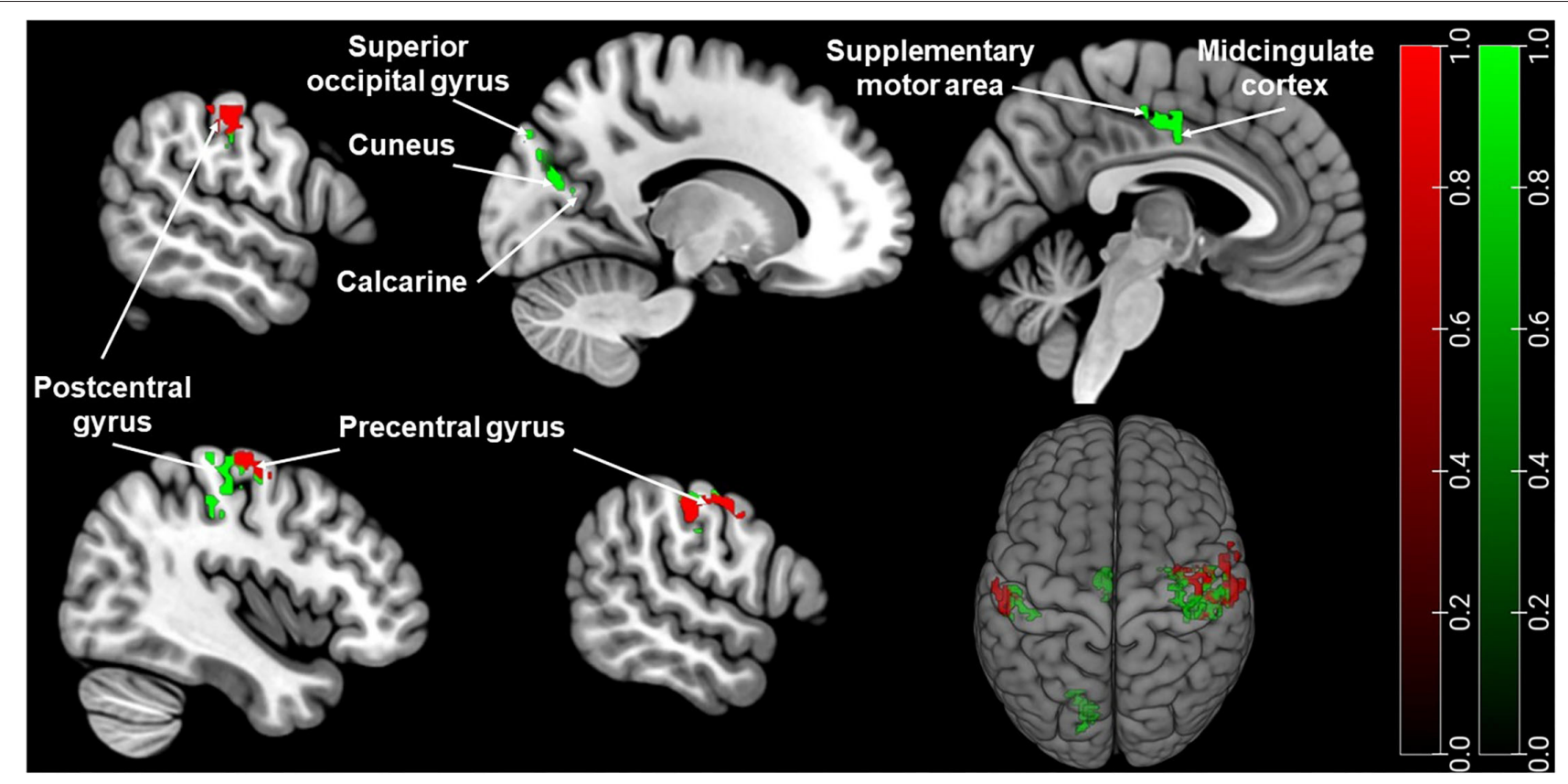

FIGURE 1 | Brain regions with significantly different PAG-FC between men and women. Green: right PAG connectivity, Red: left PAG connectivity. The significance threshold was set at cluster-level $p_{\text {FWE }}<0.05$. Coordinates are in Montreal Neurological Institute (MNI) space. For visualisation, the thresholded statistical map generated by SPM was added as an overlay image on the MNI152 brain template image in MRlcroGL (http://www.mccauslandcenter.sc.edu/mricrogl/) (31).

between the two types of MR scanners, 16 participants (eight women) were scanned in each. Among female participants, six used oral contraceptives. Among those who were not on contraceptives, six were in the luteal phase and four were in the follicular phase. There was no difference in the characteristics of the participants according to the scanner site (refer to Supplementary Table 1).

\section{Main Effects of Sex on PAG-FC}

Sex showed a significant main effect in ANOVA analysis on PAG-FC with postcentral gyrus, precentral gyrus. Sex also had a significant effect on the left PAG-FC with the inferior parietal lobe. In the case of right PAG, sex had an additional significant effect on PAG-FC with calcarine, cuneus, superior occipital gyrus, supplementary motor area (SMA), and midcingulate cortex (MCC) (refer to Figure 1 and Supplementary Table 2).

A post-hoc t-test revealed a significantly stronger PAG-FC in female migraineurs compared to males with the abovementioned regions. There was no stronger PAG-FC in males compared to females (refer to Table 2).

The type of the MR scanner had no main effect on PAG-FC and no interaction was revealed between sex and MR scanner.

\section{Migraine Characteristics and PAG-FC}

Higher migraine disability showed a significant correlation with lower PAG and superior medial part of frontal gyrus connectivity. However, there were no differences between male and female migraineurs in the effect of migraine disability on PAG-FC (see Table 3).
Average pain intensity showed no main effect, but a significant interaction with sex on PAG-FC. In male migraineurs, a significant positive correlation was found between pain intensity and PAG functional connectivity with angular gyrus, right superior parietal and occipital gyri in case of left PAG, and with angular, middle occipital, and temporal gyri in case of right PAG (refer to Table 3).

Migraine frequency did not show a significant association with PAG connectivity and there was no difference between male and female groups.

\section{Effect of Female Hormones on PAG-FC}

There were no significant differences in PAG-FC between female migraineurs using oral contraceptives compared to those who were not using. There was no significant difference in PAG-FC between women in the luteal phase compared to females in the follicular phase.

\section{DISCUSSION}

In this study, resting-state PAG functional connectivity (PAGFC) was compared between female and male migraine patients to gain new insights into sex-related differences of the "migraine brain." Some important migraine-related characteristics (attack frequency, migraine-related disability, and average pain intensity) were also taken into account to achieve a more specific exploration of the mechanisms involved in altered PAG-FC in migraine in general, and specifically, in association with sex. 
TABLE 2 | Brain regions showing a significantly stronger PAG-FC in female migraineurs compared to males.

\begin{tabular}{|c|c|c|c|c|c|}
\hline \multirow{2}{*}{$\begin{array}{l}\text { Cluster size } \\
\text { (voxel) }\end{array}$} & \multirow[t]{2}{*}{ Region } & \multicolumn{3}{|c|}{ Peak-coordinates } & \multirow{2}{*}{$\begin{array}{c}\text { Peak } \\
T \text {-value }\end{array}$} \\
\hline & & $\mathbf{x}$ & $\mathbf{y}$ & $\mathbf{z}$ & \\
\hline \multicolumn{6}{|l|}{ Left PAG } \\
\hline \multirow[t]{2}{*}{217} & R Midcingulate cortex & 4 & -12 & 46 & 6.41 \\
\hline & $\begin{array}{l}\text { R Supplementary motor } \\
\text { area }\end{array}$ & 6 & 6 & 54 & 4.85 \\
\hline \multirow[t]{2}{*}{357} & R Postcentral gyrus & 60 & -14 & 42 & 5.71 \\
\hline & R Precentral gyrus & 64 & 8 & 28 & 4.96 \\
\hline \multirow[t]{2}{*}{204} & L Inferior parietal gyrus & -56 & -20 & 50 & 5.49 \\
\hline & L Postcentral gyrus & -54 & -28 & 54 & 4.68 \\
\hline \multirow[t]{2}{*}{220} & R Precentral gyrus & 40 & -10 & 62 & 5.44 \\
\hline & R Postcentral gyrus & 48 & -20 & 60 & 4.53 \\
\hline \multicolumn{6}{|l|}{ Right PAG } \\
\hline \multirow[t]{2}{*}{859} & R Postcentral gyrus & 60 & -14 & 42 & 6.29 \\
\hline & R Precentral gyrus & 40 & -12 & 64 & 5.97 \\
\hline \multirow[t]{3}{*}{160} & L Calcarine & -20 & -70 & 14 & 5.94 \\
\hline & L Cuneus & -14 & -76 & 24 & 5.74 \\
\hline & L Superior occipital gyrus & -14 & -86 & 40 & 4.69 \\
\hline \multirow[t]{2}{*}{112} & R Calcarine & 22 & -66 & 4 & 5.62 \\
\hline & R Cuneus & 16 & -76 & 24 & 4.43 \\
\hline 202 & L Postcentral gyrus & -54 & -20 & 52 & 5.28 \\
\hline \multirow[t]{3}{*}{158} & L Paracentral_Lobule & -10 & -24 & 52 & 5.04 \\
\hline & L Supplementary motor area & -8 & -12 & 48 & 4.78 \\
\hline & L Midcingulate cortex & -4 & -6 & 38 & 4.50 \\
\hline \multirow[t]{2}{*}{111} & $\begin{array}{l}\text { R Supplementary motor } \\
\text { area }\end{array}$ & 8 & -14 & 56 & 4.79 \\
\hline & R Midcingulate cortex & 12 & -20 & 44 & 4.41 \\
\hline
\end{tabular}

Reported results are significant at cluster-level $p_{F W E}<0.05$. Coordinates are in Montreal Neurological Institute (MNI) space. R, right hemisphere; L, left hemisphere.

For the first time, we were able to identify sex-related differences in PAG-FC among migraineurs in the interictal state: female migraineurs compared to males showed a stronger resting-state PAG-FC with somatosensory and motor cortex, visual areas network (superior occipital gyrus, calcarine, cuneus), SMA and MCC. Among the measured migraine characteristics, only the average pain intensity interacted with sex on PAGFC: higher pain intensity correlated with stronger PAG-FC with middle temporal gyrus, superior occipital and parietal gyrus in male migraine patients compared to females. Migrainerelated disability is also associated with PAG-FC but without sex differences.

\section{Sex-Related Differences in PAG-FC}

Sex showed a main effect on PAG -FC with many regions of bottom-up pain processing (32). The ascending pain pathways including somatosensory and motor cortices are involved in pain perception (33), thus the increased PAG-FC with these regions may contribute to pain facilitation and central sensitisation in female migraineurs.

Further analyses revealed that PAG-FC with somatosensory and motor cortices were significantly stronger among female
TABLE 3 | Associations of migraine frequency, migraine disability, and average pain intensity with PAG-FC.

\begin{tabular}{|c|c|c|c|c|c|}
\hline \multirow{2}{*}{$\begin{array}{l}\text { Cluster size } \\
\text { (voxel) }\end{array}$} & \multirow[t]{2}{*}{ Region } & \multicolumn{3}{|c|}{ Peak-coordinates } & \multirow{2}{*}{$\begin{array}{c}\text { Peak } \\
T \text {-value }\end{array}$} \\
\hline & & $\mathbf{x}$ & y & z & \\
\hline \multicolumn{6}{|c|}{ Migraine disability } \\
\hline \multicolumn{6}{|c|}{ Negative correlation } \\
\hline \multicolumn{6}{|c|}{ Left PAG } \\
\hline 91 & $\begin{array}{l}\text { R Superior medial part of } \\
\text { frontal gyrus }\end{array}$ & 4 & 54 & 26 & 5.48 \\
\hline \multicolumn{6}{|l|}{ Right PAG } \\
\hline 109 & $\begin{array}{l}\text { R Superior medial part of } \\
\text { frontal gyrus }\end{array}$ & 4 & 54 & 26 & 5.06 \\
\hline \multicolumn{6}{|c|}{ Average pain intensity } \\
\hline \multicolumn{6}{|c|}{ Male $>$ Female } \\
\hline \multicolumn{6}{|l|}{ Left PAG } \\
\hline \multirow[t]{3}{*}{200} & R Superior occipital gyrus & 34 & -78 & 46 & 5.05 \\
\hline & R Superior parietal gyrus & 26 & -60 & 50 & 4.82 \\
\hline & R Angular gyrus & 36 & -66 & 40 & 4.72 \\
\hline \multicolumn{6}{|l|}{ Right PAG } \\
\hline \multirow[t]{3}{*}{138} & R Middle occipital gyrus & 48 & -76 & 30 & 4.06 \\
\hline & R Middle temporal gyrus & 54 & -72 & 18 & 4.03 \\
\hline & R Angular gyrus & 42 & -56 & 24 & 3.72 \\
\hline
\end{tabular}

Reported results are significant at cluster-level pFWE $<0.05$. Coordinates are in Montreal Neurological Institute (MNI) space. Migraine disability was assessed with the total score of MIDAS (Migraine Disability Assessment questionnaire). The average pain intensity of migraine attacks was measured by MIDAS-B. R, right hemisphere; L, left hemisphere.

migraineurs compared to males. Our results are in concordance with the Mainero et al. study, where they also detected increased connectivity between PAG and somatosensory and motor cortex in migraine patients who were mainly women (16). In a previous study, excitatory neurons of the somatosensory cortex were hyperexcitable in interictal women with frequent migraine attacks, in this context, stronger PAG-FC with the somatosensory cortex might make the female brain more susceptible for migraine attacks (34). Moreover, female migraineurs have thickened somatosensory cortex compared to healthy controls, but again, male results are missing (35).

Previously, in healthy females, PAG showed increased paininduced connectivity with SMA compared to men (13). SMA also has a higher likelihood of neuromagnetic activation in ictal female migraineurs during finger moving task that produces cortical hyperexcitability (36). Our results indicate stronger resting-state connectivity between PAG and SMA in pain-free female migraineurs compared to males. The stronger PAGFC with SMA may strengthen the notion of higher cortical hyperexcitability of the female brain between attacks.

In a previous study, healthy women compared to men also have stronger resting-state PAG-FC with MCC (37), involved in cognitive, affective, and attentional aspects of pain (38). Another resting-state connectivity analysis of subgenual anterior cingulate cortex (sgACC) revealed stronger connectivity between sgACC and PAG and MCC in healthy women compared to men indicating that women have a greater descending antinociceptive 
modulation contributing to more effective pain habituation compared to men (39). In migraine, a pain-induced increased activity of MCC was observed (40), however, sex differences were not assessed. Our results indicate a stronger resting-state PAGFC with MCC in pain-free female migraineurs compared to males that might contribute to the sex phenotype of migraine, but based on previous findings in healthy samples it might represent a sex-specific brain pattern rather than a disease-specific one (10).

We also found a stronger PAG-FC with visual cortex including cuneus, calcarine and superior occipital gyrus. In a previous study, these regions had a stronger paininduced PAG-FC in migraineurs compared to controls (41), and our results on sex differences might suggest that in migraine this increased connectivity might contribute to the increased interictal sensory sensitivity for females (42) that could lead to an increased vulnerability for migraine attacks (43). Impaired attentive processing of visual stimuli was also observed in female migraineurs compared to males suggesting that neurocognitive processing is affected by migraine in females (44).

Furthermore, we have to note that PAG also plays a critical role in autonomic processes, behavioural, cardiovascular, and respiratory responses to stressors and the different connectivity of PAG subregions may regulate different physiological functions $(45,46)$. The dysfunction of PAG is also implicated in anxiety (47) and depression (48), which disorders are known to be sex-differentiated and also comorbid with migraine (49).

\section{Average Pain Intensity Shows Interaction With Sex on PAG-FC}

In accordance with the general notion that migraine intensity is similar in males and females (50), our participants did not differ in average pain intensity. However, the average pain intensity of migraine attacks interacted with sex on PAG-FC in our analysis. Interestingly, increased connectivity between PAG and the angular, superior parietal, middle temporal, and superior occipital gyri correlated with higher average pain intensity only in male migraineurs.

In patients with fibromyalgia, increased activity of the angular gyrus and its stronger connectivity with pain- and analgesiarelated brain areas correlated with pain intensity analgesia after listening to music (51). In addition, the angular gyrus may exert an analgesic effect by top-down pain control during distraction or placebo analgesia (51). Indeed it has been suggested that problem-focused avoidance (cognitive and behavioural distraction) is the most frequently reported pain coping strategy for men (52).

The superior parietal, middle temporal, and occipital gyri are also involved in pain processing, their activity correlated with subjective pain in a study using a thermal heat task (53). Interestingly, these regions positively mediated the relationship between pain intensity and reported pain, however other areas such as the primary motor and the activity of the sensory cortex were uniquely related to temperature but not to pain report (53). In the study of Atlas et al., sex differences in the activity of pain mediator regions were not assessed, however, the majority of participants were male. Those previous studies in parallel with our result suggest an increased sensory sensitivity in female migraineurs (indicated by stronger PAG-FC with somatosensory cortex), meanwhile intensified subjective pain experience in male migraineurs (denoted by the correlation of average pain intensity and PAG-FC).

The PAG-FC with the above-mentioned regions may be also implicated in pain-related unpleasantness (3). Men generally show greater brain activation in pain-activated regions for both low and high pain stimuli compared to women (54). Another study demonstrated that pain intensity augments the sex differences in PAG-FC: increasing pain intensity is associated with enhanced PAG connectivity with emotion-processing areas (amygdala, caudate, and putamen) in males (13). In addition, only men showed a placebo response to pain unpleasantness, and a larger stress reduction was observed in the absence of pain among men compared to women (55). The connexion of PAG with areas of the subjective experience of pain associated with more intense migraine attacks in men in our study may suggest that they use distraction as a possible pain coping mechanism (52). This finding raises the attention to malespecific neuropathology of migraine that is less known. Migraine is underdiagnosed among males possibly because they are less willing to report pain and usually avoid going to the doctor to pretend the image of "brave man" over against "emotional woman" (56) and they are willing to use cognitive and behavioural distraction more and have higher perceived self-efficacy compared to women (57). Nonetheless, our result suggests that interindividual differences in general perceived subjective pain intensity in males might be associated with connectivity of PAG with angular, superior parietal, middle temporal, and occipital gyri.

\section{Migraine-Related Disability Correlated With PAG-FC but Did Not Interact With Sex}

In general, women are more likely to report severe headacherelated disability compared to men (58). In our study, migrainerelated disability did not differ between sexes. It was related to the FC of PAG with superior medial part of frontal gyrus (more precisely dorsomedial prefrontal cortex: dmPFC) connectivity. Specifically, higher migraine-related disability associated with decreased PAG-dmPFC connectivity indicates a weaker topdown pain control (32) without sex differences. The PFC-PAG circuitry is involved in the emotional modulation of pain (59), thus the lower PAG-FC with dmPFC in our study may suggest a weaker pain inhibition in parallel with higher migraine-related disability. A recent animal study demonstrated that the dmPFC and PAG pathway is involved in pain threshold maintenance and antianxiety behaviour, and the deactivation of $\mathrm{dmPFC}$ induces negative emotions and may lead to pain chronification (60). Our results are in concordance with this observation; however, it is not clear whether the decreased PAG-dmPFC connectivity is the cause or the consequence of increased migraine-related disability. There is a general notion about higher migraine-caused disability in women than men, but according to our questionnaire and 
fMRI results, the neuronal background of migraine disability did not show sex differences.

\section{Migraine Frequency Did Not Correlate With PAG-FC}

In general, female migraineurs have more frequent migraines than males (61). In our study, there was no difference in migraine frequency between female and male migraine groups. Migraine frequency did not correlate with PAG-FC and we found no sex differences. In previous studies, migraine frequency showed association with PAG-FC for example with SMA, prefrontal, and somatosensory cortex $(16,41)$ that we did not detect. The direct comparison with these studies is difficult because they investigated mixed migraine patients with and without aura, or patients on daily medication with high migraine frequency. However, we have examined a homogenous episodic migraine population without aura which is also a strength of the study.

\section{LIMITATIONS}

There are some limitations of our study that we should mention in this study. First, we have a small sample size in each studied group. Second, we did not have healthy control groups. Instead, we used literature results to compare our results to the sex differences in non-migraine subjects. Third, the fMRI data acquisition was assessed in two different types of MR scanners. However, we did not detect any scanner-related effects. The resolution of MR scanners could be also a potential limitation because scanning brainstem structures such as PAG is highly affected by physiological noise and the anatomical complexity of small this small region (62). In a complex preprocessing pipeline we tried to address these challenges (refer to Supplementary Figure 1). In our study, only the two sides of the PAG were used as seed regions, however, PAG connectivity may differ between subregions as well $(45,63)$. Sex differences between subregional connectivity would be an interesting direction for future studies.

Finally, female hormones might affect pain processing and PAG function $(3,17)$ thus we investigated the effect of oral contraceptives and menstrual cycle on PAG-FC. Since no differences were found, we analysed female participants together. However, in the future, it would be interesting to know whether sex hormones affect differently the functional connectivity of PAG in a larger number of migraineurs.

\section{CONCLUSION}

In this study, a stronger PAG functional connectivity with regions of ascending pain pathways in female migraineurs indicates increased brain excitability that might be a risk factor for migraine. Therefore, the PAG network may contribute to greater sensitivity and migraine vulnerability distinctively in women. However, the average pain intensity of migraine attacks exhibits stronger PAG connectivity with regions involved in the subjective experience of pain in males which may represent a consequence of increased subjective pain experience. Based on this observation, male-specific mechanisms of migraine should gain more attention. The association between other migraine characteristics and PAG connectivity seems to represent general pain-related features of migraine without sex differences. PAG connectivity shows alterations in association with migraine-related disability suggesting a decreased descending pain control. In conclusion, we were able to identify general and sex-specific differences in PAG functional connectivity among migraineurs, and thereby, show with novel results how the "migraine brain" may be differently affected in females and males even in the interictal state.

\section{AUTHOR'S NOTE}

Preliminary data from this study were presented at the following event: 34th ECNP Congress 2-5 October 2021, Lisbon, Portugal poster presentation.

\section{DATA AVAILABILITY STATEMENT}

The datasets presented in this article are not readily available because they contain information that could compromise the privacy of research participants. Requests to access the datasets should be directed to juhasz.gabriella@pharma.semmelweis-univ.hu.

\section{ETHICS STATEMENT}

The studies involving human participants were reviewed and approved by Scientific and Research Ethics Committee of the Medical Research Council (Hungary). The patients/participants provided their written informed consent to participate in this study.

\section{AUTHOR CONTRIBUTIONS}

The study was designed and conceived by GJ and GK. ES, $\mathrm{NK}$, and AG were responsible for subject recruitment and data collection. Data analysis was performed by KG with assistance from GJ, GK, CSA, and ME. GB, DD, DB, and GJ contributed to the interpretation to the data. $\mathrm{KG}, \mathrm{DB}$, and GJ wrote the first draught of the manuscript. All authors contributed to the article and approved the submitted version.

\section{FUNDING}

This study was supported by the Hungarian Academy of Sciences (MTA-SE Neuropsychopharmacology and Neurochemistry Research Group); the Hungarian Brain Research Program (Grants: 2017-1.2.1-NKP-2017-00002; KTIA_13_NAPA-II/14); 
the National Development Agency (Grant: KTIA_NAP_13-12013- 0001); by the Hungarian Academy of Sciences, Hungarian National Development Agency, Semmelweis University and the Hungarian Brain Research Program (Grant: KTIA_NAP_13-22015-0001) (MTA-SE-NAP B Genetic Brain Imaging Migraine Research Group); by the Thematic Excellence Programme (Tématerületi Kiválósági Program, 2020-4.1.1.-TKP2020) of the Ministry for Innovation and Technology in Hungary, within the framework of the Neurology and Translational Biotechnology thematic programmes of the Semmelweis University; by the National Research, Development and Innovation Office, Hungary (2019-2.1.7-ERA-NET-2020-00005), under the frame of ERA PerMed (ERAPERMED2019-108); and by the UNKP20-3-II-SE-51 New National Excellence Program of the Ministry for Innovation and Technology from the source of the National

\section{REFERENCES}

1. Steiner TJ, Stovner LJ, Jensen R, Uluduz D, Katsarava Z. Lifting the burden: the global campaign against $\mathrm{H}$. Migraine remains second among the world's causes of disability, and first among young women: findings from GBD2019. J Headache Pain. (2020) 21:137. doi: 10.1186/s10194-020-01208-0

2. Lagman-Bartolome AM, Lay C. Migraine in women. Neurol Clin. (2019) 37:835-45. doi: 10.1016/j.ncl.2019.07.002

3. Choi Jae C, Park Sang K, Kim Y-H, Shin Y-W, Kwon Jun S, Kim Jin S, et al. Different brain activation patterns to pain and pain-related unpleasantness during the menstrual cycle. Anesthesiology. (2006) 105:120-7. doi: 10.1097/00000542-200607000-00021

4. Borsook D, Erpelding N, Lebel A, Linnman C, Veggeberg R, Grant PE, et al. Sex and the migraine brain. Neurobiol Dis. (2014) 68:200-14. doi: 10.1016/j.nbd.2014.03.008

5. van Casteren DS, Verhagen IE, Onderwater GL, MaassenVanDenBrink A, Terwindt GM. Sex differences in prevalence of migraine trigger factors: a cross-sectional study. Cephalalgia. (2021) 41:643-8. doi: $10.1177 / 0333102420974362$

6. Alebna P, Maleki N. Allostatic load in perimenopausal women with migraine. Front Neurol. (2021) 12:649423. doi: 10.3389/fneur.2021.649423

7. Maleki N, Becerra L, Borsook D. Migraine: maladaptive brain responses to stress. Headache. (2012) 52(Suppl.2):102-6. doi: 10.1111/j.1526-4610.2012.02241.x

8. Maleki N, Androulakis XM. Is there any MRI pattern that discriminates female from male migraine patients? Front Neurol. (2019) 10:961. doi: 10.3389/fneur.2019.00961

9. Dai Z, Zhong J, Xiao P, Zhu Y, Chen F, Pan P, et al. Gray matter correlates of migraine and gender effect: a meta-analysis of voxel-based morphometry studies. Neuroscience. (2015) 299:88-96. doi: 10.1016/j.neuroscience.2015.04.066

10. Maleki N, Linnman C, Brawn J, Burstein R, Becerra L, Borsook D. Her vs. his migraine: multiple sex differences in brain function and structure. Brain. (2012) 135:2546-59. doi: 10.1093/brain/aws175

11. Szabó E, Galambos A, Kocsel N, Édes AE, Pap D, Zsombók T, et al. Association between migraine frequency and neural response to emotional faces: an fMRI study. Neuroimage Clin. (2019) 22:101790. doi: 10.1016/j.nicl.2019.101790

12. Zhang J, Su J, Wang M, Zhao Y, Zhang QT, Yao Q, et al. The posterior insula shows disrupted brain functional connectivity in female migraineurs without aura based on brainnetome atlas. Sci Rep. (2017) 7:16868. doi: 10.1038/s41598-017-17069-8

13. Linnman C, Beucke JC, Jensen KB, Gollub RL, Kong J. Sex similarities and differences in pain-related periaqueductal gray connectivity. Pain. (2012) 153:444-54. doi: 10.1016/j.pain.2011.11.006

14. Knight YE, Goadsby PJ. The periaqueductal grey matter modulates trigeminovascular input: a role in migraine? Neuroscience. (2001) 106:793800. doi: 10.1016/S0306-4522(01)00303-7
Research, Development and Innovation Fund. The sponsors had no role in the design of the study, in the collection, analysis, interpretation of data, and in the writing of the manuscript.

\section{ACKNOWLEDGMENTS}

The authors extend their gratitude to Andrea Edit Édes, Zsuzsanna Tóth, Dorka Papp, Terézia Zsombók, Ádám György Békésy-Szabó, and Lajos Rudolf Kozák for their contribution.

\section{SUPPLEMENTARY MATERIAL}

The Supplementary Material for this article can be found online at: https://www.frontiersin.org/articles/10.3389/fpain. 2021.767162/full\#supplementary-material

15. Weiller C, May A, Limmroth V, Jüptner M, Kaube H, Schayck RV, et al. Brain stem activation in spontaneous human migraine attacks. Nat Med. (1995) 1:658-60. doi: 10.1038/nm0795-658

16. Mainero C, Boshyan J, Hadjikhani N. Altered functional magnetic resonance imaging resting-state connectivity in periaqueductal gray networks in migraine. Ann Neurol. (2011) 70:838-45. doi: 10.1002/ana. 22537

17. Wang D, Zhao J, Wang J, Li J, Yu S, Guo X. Deficiency of female sex hormones augments PGE2 and CGRP levels within midbrain periaqueductal gray. $J$ Neurol Sci. (2014) 346:107-11. doi: 10.1016/j.jns.2014.08.002

18. Sheehan DV, Lecrubier Y, Sheehan KH, Amorim P, Janavs J, Weiller E, et al. The Mini-International Neuropsychiatric Interview (M.I.N.I.): the development and validation of a structured diagnostic psychiatric interview for DSM-IV and ICD-10. J Clin Psychiatry. (1998) 59(Suppl.20):22-33.

19. Headache Classification Committee of the International Headache Society (IHS). The International Classification of Headache Disorders, 3rd edition (beta version). Cephalalgia. (2013) 33:629-808. doi: $10.1177 / 0333102413485658$

20. Oldfield RC. The assessment and analysis of handedness: the Edinburgh inventory. Neuropsychologia. (1971) 9:97-113. doi: 10.1016/0028-3932(71)90067-4

21. Stewart WF, Lipton RB, Kolodner KB, Sawyer J, Lee C, Liberman JN. Validity of the Migraine Disability Assessment (MIDAS) score in comparison to a diary-based measure in a population sample of migraine sufferers. Pain. (2000) 88:41-52. doi: 10.1016/S0304-3959(00)00305-5

22. Dale AM, Fischl B, Sereno MI. Cortical surface-based analysis. I Segmentation and surface reconstruction. Neuroimage. (1999) 9:179-94. doi: 10.1006/nimg.1998.0395

23. Grabner G, Janke AL, Budge MM, Smith D, Pruessner J, Collins DL. Symmetric atlasing and model based segmentation: an application to the hippocampus in older adults. Medical Image Computing Comput Assist Interv. (2006) 9:58-66. doi: 10.1007/11866763_8

24. Avants BB, Epstein CL, Grossman M, Gee JC. Symmetric diffeomorphic image registration with cross-correlation: evaluating automated labeling of elderly and neurodegenerative brain. Med Image Anal. (2008) 12:26-41. doi: 10.1016/j.media.2007.06.004

25. Jenkinson M, Bannister P, Brady M, Smith S. Improved optimization for the robust and accurate linear registration and motion correction of brain images. Neuroimage. (2002) 17:825-41. doi: 10.1006/nimg.2002.1132

26. Behzadi Y, Restom K, Liau J, Liu TT. A component based noise correction method (CompCor) for BOLD and perfusion based fMRI. Neuroimage. (2007) 37:90-101. doi: 10.1016/j.neuroimage.2007.04.042

27. Smith SM, Brady JM. SUSAN-a new approach to low level image processing. Int J Comput Vis. (1997) 23:45-78. doi: 10.1023/A:1007963824710

28. Pruim RHR, Mennes M, van Rooij D, Llera A, Buitelaar JK, Beckmann CF, et al. A robust ICA-based strategy for removing motion artifacts from fMRI data. Neuroimage. (2015) 112:267-77. doi: 10.1016/j.neuroimage.2015.02.064 
29. Friston KJ, Williams S, Howard R, Frackowiak RS, Turner R. Movementrelated effects in fMRI time-series. Magnet Resonance Med. (1996) 35:346-55. doi: 10.1002/mrm.1910350312

30. Worsley KJ, Chen JI, Lerch J, Evans AC. Comparing functional connectivity via thresholding correlations and singular value decomposition. Philos Trans R Soc Lond B Biol Sci. (2005) 360:913-20. doi: 10.1098/rstb.2005.1637

31. Rorden C, Brett M. Stereotaxic display of brain lesions. Behav Neurol. (2000) 12:191-200. doi: 10.1155/2000/421719

32. Schweinhardt P, Bushnell MC. Pain imaging in health and disease-how far have we come? J Clin Invest. (2010) 120:3788-97. doi: 10.1172/JCI43498

33. Apkarian AV, Bushnell MC, Treede RD, Zubieta JK. Human brain mechanisms of pain perception and regulation in health and disease. Eur $J$ Pain. (2005) 9:463-84. doi: 10.1016/j.ejpain.2004.11.001

34. Lang E, Kaltenhäuser M, Neundörfer B, Seidler S. Hyperexcitability of the primary somatosensory cortex in migraine-a magnetoencephalographic study. Brain. (2004) 127:2459-69. doi: 10.1093/brain/awh295

35. Kim JH, Kim JB, Suh SI, Seo WK, Oh K, Koh SB. Thickening of the somatosensory cortex in migraine without aura. Cephalalgia. (2014) 34:112533. doi: $10.1177 / 0333102414531155$

36. Ge HT, Liu HX, Xiang J, Miao AL, Tang L, Guan QS, et al. Abnormal cortical activation in females with acute migraine: a magnetoencephalography study. Clin Neurophysiol. (2015) 126:170-9. doi: 10.1016/j.clinph.2014.03.033

37. Kong J, Tu PC, Zyloney C, Su TP. Intrinsic functional connectivity of the periaqueductal gray, a resting fMRI study. Behav Brain Res. (2010) 211:215-9. doi: 10.1016/j.bbr.2010.03.042

38. Vogt BA. Midcingulate cortex: structure, connections, homologies, functions and diseases. J Chem Neuroanat. (2016) 74:28-46. doi: 10.1016/j.jchemneu.2016.01.010

39. Wang G, Erpelding N, Davis KD. Sex differences in connectivity of the subgenual anterior cingulate cortex. Pain. (2014) 155:755-63. doi: 10.1016/j.pain.2014.01.005

40. Schwedt TJ, Chong CD, Chiang CC, Baxter L, Schlaggar BL, Dodick DW. Enhanced pain-induced activity of pain-processing regions in a case-control study of episodic migraine. Cephalalgia. (2014) 34:947-58. doi: $10.1177 / 0333102414526069$

41. Solstrand Dahlberg L, Linnman CN, Lee D, Burstein R, Becerra L, Borsook D. Responsivity of periaqueductal gray connectivity is related to headache frequency in episodic migraine. Front Neurol. (2018) 9:61. doi: 10.3389/fneur.2018.00061

42. Zhou ZY Yu YW, Wu D, Liu HX, Xiang J, Wu T, et al. Abnormality of visual neuromagnetic activation in female migraineurs without aura between attacks. J Headache Pain. (2019) 20:7. doi: 10.1186/s10194-018-0957-9

43. Schwedt TJ. Multisensory integration in migraine. Curr Opin Neurol. (2013) 26:248-53. doi: 10.1097/WCO.0b013e328360edb1

44. Guo Y, Xu S, Nie S, Han M, Zhang Y, Chen J, et al. Female vs. male migraine: an event-related potential study of visual neurocognitive processing. J Headache Pain. (2019) 20:38. doi: 10.1186/s10194-019-0995-y

45. Coulombe MA, Erpelding N, Kucyi A, Davis KD. Intrinsic functional connectivity of periaqueductal gray subregions in humans. Hum Brain Mapp. (2016) 37:1514-30. doi: 10.1002/hbm.23117

46. Dampney RA, Furlong TM, Horiuchi J, Iigaya K. Role of dorsolateral periaqueductal grey in the coordinated regulation of cardiovascular and respiratory function. Auton Neurosci. (2013) 175:17-25. doi: 10.1016/j.autneu.2012.12.008

47. Donner NC, Lowry CA. Sex differences in anxiety and emotional behavior. Pflugers Arch. (2013) 465:601-26. doi: 10.1007/s00424-013-1271-7

48. Ho YC, Lin TB, Hsieh MC, Lai CY, Chou D, Chau YP, et al. Periaqueductal gray glutamatergic transmission governs chronic stress-induced depression. Neuropsychopharmacology. (2018) 43:302-12. doi: 10.1038/npp.2017.199

49. Buse DC, Reed ML, Fanning KM, Bostic R, Dodick DW, Schwedt TJ, et al. Comorbid and co-occurring conditions in migraine and associated risk of increasing headache pain intensity and headache frequency: results of the migraine in America symptoms and treatment (MAST) study. J Headache Pain. (2020) 21:23. doi: 10.1186/s10194-020-1084-y
50. Al-Hassany L, Haas J, Piccininni M, Kurth T, Maassen Van Den Brink A, Rohmann JL. Giving researchers a headache - sex and gender differences in migraine. Front Neurol. (2020) 11:549038. doi: 10.3389/fneur.2020.549038

51. Garza-Villarreal EA, Jiang Z, Vuust P, Alcauter S, Vase L, Pasaye EH, et al. Music reduces pain and increases resting state fMRI BOLD signal amplitude in the left angular gyrus in fibromyalgia patients. Front Psychol. (2015) 6:1051. doi: 10.3389/fpsyg.2015.01051

52. Unruh AM, Ritchie J, Merskey H. Does gender affect appraisal of pain and pain coping strategies? Clin J Pain. (1999) 15:31-40. doi: 10.1097/00002508-199903000-00006

53. Atlas LY, Lindquist MA, Bolger N, Wager TD. Brain mediators of the effects of noxious heat on pain. Pain. (2014) 155:1632-48. doi: 10.1016/j.pain.2014.05.015

54. Kong J, Loggia ML, Zyloney C, Tu P, LaViolette P, Gollub RL. Exploring the brain in pain: activations, deactivations and their relation. Pain. (2010) 148:257-67. doi: 10.1016/j.pain.2009.11.008

55. Aslaksen PM, Bystad M, Vambheim SM, Flaten MA. Gender differences in placebo analgesia: event-related potentials and emotional modulation. Psychosom Med. (2011) 73:193-9. doi: 10.1097/PSY.0b013e31820 $80 \mathrm{~d} 73$

56. Robinson ME, Riley JL 3rd, Myers CD, Papas RK, Wise EA, Waxenberg LB, et al. Gender role expectations of pain: relationship to sex differences in pain. J Pain. (2001) 2:251-7. doi: 10.1054/jpai.2001.24551

57. Bartley EJ, Fillingim RB. Sex differences in pain: a brief review of clinical and experimental findings. Br J Anaesth. (2013) 111:52-8. doi: 10.1093/bja/ aet127

58. Lipton RB, Munjal S, Alam A, Buse DC, Fanning KM, Reed ML, et al. Migraine in America Symptoms and Treatment (MAST) study: baseline study methods, treatment patterns, and gender differences. Headache. (2018) 58:1408-26. doi: 10.1111/head.13407

59. Bushnell MC, Ceko M, Low LA. Cognitive and emotional control of pain and its disruption in chronic pain. Nat Rev Neurosci. (2013) 14:502-11. doi: 10.1038/nrn3516

60. Yin JB, Liang SH, Li F, Zhao WJ, Bai Y, Sun Y, et al. dmPFC-vlPAG projection neurons contribute to pain threshold maintenance and antianxiety behaviors. J Clin Invest. (2020) 130:6555-70. doi: 10.1172/JCI127607

61. Finocchi C, Strada L. Sex-related differences in migraine. Neurol Sci. (2014) 35(Suppl.1):207-13. doi: 10.1007/s10072-014-1772-y

62. Beissner F. Functional MRI of the brainstem: common problems and their solutions. Clin Neuroradiol. (2015) 25(Suppl.2):251-7. doi: 10.1007/s00062-015-0404-0

63. Satpute AB, Wager TD, Cohen-Adad J, Bianciardi M, Choi JK, Buhle JT, et al. Identification of discrete functional subregions of the human periaqueductal gray. Proc Natl Acad Sci USA. (2013) 110:17101-6. doi: $10.1073 /$ pnas. 1306095110

Conflict of Interest: The authors declare that the research was conducted in the absence of any commercial or financial relationships that could be construed as a potential conflict of interest.

Publisher's Note: All claims expressed in this article are solely those of the authors and do not necessarily represent those of their affiliated organizations, or those of the publisher, the editors and the reviewers. Any product that may be evaluated in this article, or claim that may be made by its manufacturer, is not guaranteed or endorsed by the publisher.

Copyright (C) 2021 Gecse, Baksa, Dobos, Aranyi, Galambos, Kocsel, Szabó, Kökönyei, Emri, Bagdy and Juhasz. This is an open-access article distributed under the terms of the Creative Commons Attribution License (CC BY). The use, distribution or reproduction in other forums is permitted, provided the original author(s) and the copyright owner(s) are credited and that the original publication in this journal is cited, in accordance with accepted academic practice. No use, distribution or reproduction is permitted which does not comply with these terms. 